\title{
Expect The Unexpected: A Qualitative Study of The Ripple Effects of Children's Mental Health Services Implementation Efforts
}

\section{Michael D. Pullmann ( $\nabla$ pullmann@uw.edu )}

University of Washington School of Medicine https://orcid.org/0000-0001-8404-864X

Shannon Dorsey

University of Washington School of Medicine

Mylien T. Duong

Impact Research And Development Organisation

Aaron R. Lyon

University of Washington School of Medicine

lan Muse

University of Washington

Cathy M. Corbin

University of Washington School of Medicine

Chayna J. Davis

University of Washington School of Medicine

Kristin Thorp

Youth MOVE National

Millie Sweeney

FREDLA

Cara C. Lewis

Kaiser Permanente Washington Health Research Institute

Byron J. Powell

Brown School at the University of Washington St. Louis

\section{Research}

Keywords: Ripple effects, Children's mental health services, Implementation strategies, Unintended consequences

Posted Date: July 8th, 2021

DOI: https://doi.org/10.21203/rs.3.rs-595020/v1 
License: (c) (i) This work is licensed under a Creative Commons Attribution 4.0 International License. Read Full License 


\section{Abstract}

Background: Strategies to implement evidence-based interventions (EBIs) in children's mental health services have complex direct and indirect causal impacts on multiple outcomes. Ripple effects as outcomes that are caused by EBI implementation efforts and are indirect, unplanned, unanticipated, and/or more salient to stakeholders other than to researchers and implementers. The purpose of the current paper is to provide a compilation of possible ripple effects associated with EBI implementation strategies in children's mental health services, to be used for treatment development, implementation planning, research, and quality improvement.

Methods: Participants were identified via expert nomination and snowball sampling. Online surveys were completed by 81 participants representing one of five roles: providers of mental health services to children or youth, researchers, policy makers, caregiver, and youth. Participants brainstormed possible ripple effects. A partially directed conventional content analysis with consensus decision making was used to code ripple effects.

Results: Four hundred and four unique responses were coded into 66 ripple effects and 14 categories. Example categories include general knowledge, skills, attitudes, and confidence about using EBI's; general job-related ripple effects; EBI treatment adherence, fidelity, and alignment; gaming the system; equity and stigma; shifting roles, role clarity, and task shifting; economic costs and benefits; EBI treatment availability, access, participation, attendance, barriers, and facilitators; clinical process and treatment quality; client engagement, therapeutic alliance, and client satisfaction; clinical organization structure, relationships in the organization, process, and functioning; youth client and caregiver outcomes; and use of EBI strategies and insights in one's own life.

Conclusions: This research advances the field by providing implementers, researchers, funders, policy makers, and consumers with a "menu" of potential ripple effects for consideration when evaluating the full balance of any implementation effort. It can be a practical tool to ensure compliance with the guidance of experts from Quality Improvement/Quality Assurance, Complexity Science, Diffusion of Innovation Theory, and the Medical Research Council. Future phases will match potential ripple effects with the most salient implementation strategies for each participant role.

Trial Registration: None.

\section{Contributions To The Literature}

- This qualitative study of multiple stakeholders in children's mental health services identifies several possible ripple effects of implementation strategies, opening a new area of study for implementation science.

- Ripple effects can contribute a positive, negative, or neutral accounting when considering the full balance of implementation quality and impact. 
- The list of ripple effects will provide implementation scientists, developers, and others with a useful tool during implementation planning and evaluation.

- This expert-informed methodology can provide a model for other fields for exploring possible ripple effects within implementation science.

\section{Introduction}

Closing the science-to-practice gap in children's mental health services is of enormous public health significance. Mental health $(\mathrm{MH})$ disorders are the most costly health conditions of childhood, affecting one out of every five children in the United States. ${ }^{1}$ Over 650 Evidence Based Interventions (EBIs) have been developed to treat child $\mathrm{MH}$ problems, ${ }^{2,3}$ but very few youth in community mental health settings receive them. ${ }^{4,5}$ Implementation science aims to close this science-to-practice gap, with significant progress in operationalizing implementation strategies and outcomes. There have been considerable advances in implementation science methods to consistently measure and report implementation strategies, ${ }^{6-9}$ and measures of implementation-specific outcomes. ${ }^{10}$ The Expert Recommendations for Implementing Change study refined a compilation of 73 discrete implementation strategies, ${ }^{11,12}$ and the compilation has recently been adapted to school mental health contexts. ${ }^{13,14}$

Implementation strategies have complex direct and indirect causal impacts on multiple outcomes, and these impacts vary as a function of context, needs, and stakeholders. Implementation strategies are often selected based on what those who are planning the implementation (for example, intermediary organizations, service providers, and EBI purveyors) have done in the past or what is readily available. ${ }^{15}$ Implementation scientists and intermediary organizations have developed approaches to enable a systematic process of identifying mechanisms and causal processes that underlie implementation strategies. ${ }^{15-18}$ This work has focused on primary implementation outcomes, but is limited in its focus on potential unintended outcomes that could result from implementation efforts.

Implementation efforts are primarily focused on the highly critical outcomes of adoption and delivery of the intervention with fidelity; ${ }^{19-21}$ other primary implementation outcomes include acceptability, appropriateness, feasibility, penetration, implementation cost, and sustainability. ${ }^{10}$ However, any change in complex systems such as health care can lead to a cascading array of unplanned, non-linear, or unexpected "ripple" effects. We define ripple effects as outcomes that are caused by EBI implementation efforts and are indirect, unplanned, unanticipated, and/or more salient to stakeholders other than to researchers and implementers. The possibility of ripple effects is rarely considered when planning implementation in a research context, as these outcomes are distal and indirect, or are more salient to nonresearcher stakeholders. Identifying, measuring, planning for, and addressing ripple effects should be a priority for implementation science, as ripple effects can hinder or enhance implementation outcomes, effectiveness, and sustainability. ${ }^{22}$ The purpose of the current paper is to provide all types of implementers-intermediary organizations, purveyors, mental health providers, researchers, and treatment 
developers-with a compilation of possible ripple effects associated with implementation of EBI in children's mental health services.

Ripple effects include distal and indirect outcomes on not only the child/youth receiving mental health services, but also on other individuals and subjects other than child health, such as caregiver strain or the dynamics of health care teams. Ripple effects also include indirect mediators or process variables not believed to be central to the mechanism of effectiveness (e.g., clinician sense of autonomy). What defines a construct as a ripple effect is not the construct itself, but its place in the overall theoretical causal model of the implementation strategy. Constructs that are intended mediators or outcomes in the context of one study or implementation effort may be ripple effects in a different study or implementation effort. Even client symptoms and functioning may be a ripple effect, if unintended and/or distally connected in the causal chain. As an example, fidelity of treatment delivery is an intended outcome of many implementation strategies, such as training. However, fidelity of treatment delivery may be a ripple effect for implementation strategies not focused on fidelity, such as state mandates requiring EBP treatment delivery. Mandates have actually been found to decrease fidelity while increasing adoption, penetration, and reach. ${ }^{23}$ In this example, fidelity is not an intended outcome but is a ripple effect.

As with any outcome, ripple effects are likely to be associated with both the type and quality of the causal action. In other words, the quality of implementation strategy delivery likely moderates the valence of the ripple effect. We would expect that higher quality implementation efforts are more likely to lead to more positive ripple effects. As an example, peer-to-peer support is one implementation strategy to help youth with mental health challenges engage with and successfully complete effective mental health treatment services. When peer-to-peer support is implemented with high-quality training and supervision, peers may experience high job satisfaction and empowerment. When peer-to-peer support is implemented more poorly, peer support providers may experience lower job satisfaction and burnout.

Ripple effects are already recognized as important outside of implementation science. For example, the Medical Research Council's guidance on process evaluation of complex interventions identified unexpected mechanisms and outcomes as an area worthy of investigation. ${ }^{24}$ Ripple effects are central to Quality Improvement and Quality Assurance (QI/QA), ${ }^{25}$ Complexity Science, ${ }^{22}$ and Diffusion of Innovations theory. ${ }^{21} \mathrm{QI} / \mathrm{QA}$ refers to ripple effects as "Balancing Measures," as they are essential for understanding the full balance of costs and benefits of an action. Changes in one part of a system may cause problems or improvements in other parts of the system. ${ }^{25,26} \mathrm{QI} / \mathrm{QA}$ also recognizes that all outcomes are relative to the person or systems that are impacted, and therefore it is essential to consider a wide variety of viewpoints in the outcome measure selection process.

However, attention to ripple effects has been extremely limited. Two systematic reviews conducted 25 years apart found fewer than $0.2 \%$ of studies on innovations in a broad range of fields examined whether the innovation resulted in any unplanned outcomes; ${ }^{21,27}$ other reviews have found similar absence in perioperative care improvement interventions, ${ }^{28}$ improvement methodologies in surgery, ${ }^{29}$ and interventions to reduce patient falls and infections. ${ }^{30}$ Unplanned and unintended ripple effects are 
infrequently measured even when using strategies specifically designed to consider the full range of implementation outcomes. Complexity science, for example, specifically accepts that unintended consequences are a normal part of any system intervention and emphasizes the importance of intentionally examining them. However, a scoping review of 22 studies that used a complexity science informed intervention design found only $50 \%$ featured an intentional process to identify unintended or negative consequences. ${ }^{22}$

The ripple effects of implementation strategies in children's mental health services are rarely examined. For example, in a recent systematic review of 18 studies intended to assess the effectiveness and harms of children's $\mathrm{MH}$ implementation strategies, the authors identified only one study that systematically investigated whether there actually were potential harms. ${ }^{31}$ This study, which examined the impact of professional training on client identification and referral rates for first episode psychosis, found impact on two possible ripple effects: adverse events and false-positive identification. ${ }^{32}$ Other relevant studies also illustrate that ripple effects can be positive or negative, and that ripple effects may result from an interaction of the implementation strategy and intervention. In a randomized trial of the effectiveness of an intervention to reduce child neglect, researchers used an intervention $\mathrm{x}$ implementation strategy $2 \times 2$ factorial design (Intervention: EBI vs. services as usual; Implementation strategy: fidelity monitoring and feedback vs. no fidelity monitoring and feedback). Results indicated that providing fidelity monitoring and feedback along with the provision of the EBI was associated with greater staff retention; ${ }^{33}$ however, providing fidelity monitoring and feedback to the services as usual group was associated with greater staff emotional exhaustion. ${ }^{34}$ In other words, the same implementation strategy in the same study had different ripple effects, one positive (retention) and one negative (emotional exhaustion), depending on the intervention condition. Contradictory ripple effects may also emerge along with intended findings. As mentioned earlier, Park et al. (2018) ${ }^{23}$ examined the impact of a state policy encouraging EBI adoption. While the policy resulted in increased EBI adoption and reach, it also increased negative ripple effects such as low-quality EBI use, "off-label" treatment, and modified, low-fidelity or "counterfeit" versions of EBIs. ${ }^{35}$ Studying ripple effects highlights their causal complexity, while providing direction for implementation efforts to tailor implementation strategies and thereby improve strategy usability and beneficial outcomes.

The reasons for the relative lack of attention to ripple effects are varied. Over eighty years ago, Robert Merton first described several factors that limit human beings' ability to consider and anticipate consequences, four of which are pertinent here: (a) general lack of foreknowledge (not knowing what you do not know); (b) implicit assumptions and assessment errors in strategy selection; (c) the "impervious immediacy of interest," also known as "tunnel vision" or "innovation bias" (a term coined by Everett Rogers in Diffusion of Innovations Theory), caused by an intense focus on desired immediate consequences, such as a strong desire for positive results, so that other consequences are not considered; and (d) decision making solely based on values in the absence of logic or rationale. ${ }^{21,36}$ Sometimes, ripple effects may be negative unintended consequences that could dampen enthusiasm for specific implementation efforts. Because research is often driven by implementers and others who have a stake in the outcomes, findings that are not supportive might be more likely to be quashed or minimized. 
Sveiby and colleagues conducted a survey of researchers who study unintended consequences to uncover their beliefs about why others do not study unintended consequences more often. ${ }^{27}$ Consistent with the work of Robert Merton and Everett Rogers, ${ }^{20,21,37}$ participants rated pro-innovation bias as the principal reason unintended consequences are rarely included. The second ranked reason was a belief that funders are less interested in research on unintended consequences-also a form of innovation bias. As Sveiby points out, these two reasons are political and emotional in nature, ${ }^{27}$ and do not positively serve the interests of science, quality implementation, or quality improvement. Ignoring ripple effects does not make them go away ${ }^{38}$ Importantly, participants did not believe methodological difficulties were barriers to this work, particularly if reliable methods for anticipating and measuring ripple effects existed. Improving our methods of studying ripple effects may result in increased acceptance and inclusion.

\section{Purpose of the Current Study}

The goal of the current manuscript is to develop a compilation of possible implementation ripple effects from the viewpoint of primary stakeholders of children's mental health services (youth receiving services, their caregivers, mental health providers, policy makers, and researchers) to support implementation decision-making by anticipating and detecting ripple effects. We do not, however, intend to present a checklist of ripple effects that must be examined in all implementation efforts, as this would be unwieldy and infeasible. We hope that the compilation we provide might temper the factors described by Merton, Rogers, and Sveiby in the following ways. It would reduce lack of awareness by explicitly describing ripple effects in order to facilitate their consideration and inclusion during research and implementation planning efforts. It may also reduce tunnel vision and innovation bias by normalizing the inclusion of ripple effects as an area of study. Once detected during implementation efforts, desirable ripple effects can be capitalized upon, and undesirable ripple effects can be ameliorated, thereby contributing to quality assurance and improvement. Our intent is to describe the range of possible ripple effects to consider for implementation in children's mental health, which also may serve as a model for other fields.

\section{Method}

This qualitative research study featured an open-ended coding of survey responses into multiple themes, under the assumptions of a post-positivist paradigm. This paradigm was applied because the purpose of the research was to obtain a wide range of possible ripple effects that are salient to multiple participant types. The research team, who have deep experience in children's mental health research and implementation, assumed that the validity and transferability of the coding process was inherently tied to their own perceptions and context. The research team's characteristics as cisgender, white academics within a large, traditional medical school, likely contributed to the ripple effect categories. As described, we made several efforts to obtain diverse participants in terms of role, geography, and race/ethnicity, in order to better obtain broad perspectives. While another team may have identified or classified these codes differently, the team believes our conclusions point towards deeper truths that are useful for other researchers and implementers. 


\section{Participants}

Table 1 provides sample demographics. The sample consisted of a total $N=81$ participants, $n=23$ (28.4\%) providers of mental health services to children and youth, $n=14(17.3 \%)$ researchers, $n=6(7.4 \%)$ policy makers, $n=18(22.2 \%)$ caregivers of youth consumers of mental health services, and $n=20(24.7 \%)$ youth consumers of mental health services. A majority of participants were female and White. We did not collect age, but $6.2 \%$ participants were under 18 years.

Table 1 Demographics of Full Population 


\begin{tabular}{|lll|}
\hline Baseline demographics & $n$ & $\%$ \\
\hline Age & & \\
$>18$ Years of Age & 76 & 93.8 \\
$<18$ Years of Age & 5 & 6.2 \\
Gender & & \\
Female & 63 & 77.8 \\
Male & 15 & 18.5 \\
Something Else & 3 & 3.7 \\
\hline Race & & \\
American Indian or Alaskan Native & 3 & 3.7 \\
Asian & 10 & 12.3 \\
Black & 6 & 7.4 \\
Native Hawaiian or Pacific Islander & 0 & 0.0 \\
White & 51 & 63.0 \\
Other & 7 & 8.6 \\
Prefer not to say & 4 & 4.9 \\
\hline Ethnicity & 20 & 24.7 \\
Latinx & 11 & 13.6 \\
Non-Latinx & 70 & 86.4 \\
\hline Role & 6 & 7.4 \\
Provider & 18 & 22.2 \\
Researcher & 28.4 \\
Policymaker & & \\
Caregiver & & \\
Youth & & \\
\hline
\end{tabular}

\section{Procedures}

Participants were recruited from throughout the United States using purposeful sampling to ensure diversity, representativeness, and expertise within the five roles. Criteria for participation were that participants had to (a) fit their role (i.e. be a caregiver of a child/youth who received mental health services; a youth aged 16-25 who have received mental health services; a youth mental health therapist; a policy maker such as a state, county, or regional mental health director, legislator, or legislative staff; or a 
researcher), and (b) have experience with some aspect of implementing children's mental health services and supports, such as working on mental health policy, advisory committees, providing peer support, and conducting training. Participants were identified with the support of several organizations. We aimed to recruit approximately 20 participants from each role and generally met this goal except for policy makers. As described in the results, we found that our recruitment led to code saturation. However, we struggled with recruitment from policy makers. Youth consumers were identified by Youth Motivating Others through Voices of Experience (Youth MOVE National), which did direct outreach to invite 40 youth to participate, and when this did not identify enough youth, sent announcements on Twitter, Facebook, and an internal email list. Caregivers were recruited by the Family-Run Executive Director Leadership Association (FREDLA), which initially reached out directly to approximately 35 family leaders, and when this did not identify enough caregivers, emailed a recruitment flyer to a listserv. Researchers and providers were identified via nominations by the study team and colleagues of the team from the School Mental Health Assessment Research and Training (SMART) Center and via an email to listservs run by the Society for Implementation Research Collaboration (SIRC). Policy makers were recruited via warm handoff emails from colleagues of the research team, and cold-emails to addresses identified via contacts provided by the National Association of State Mental Health Program Directors (NASMHPD). Additionally, our research and consultant team brainstormed a list of researchers, providers, and policy makers with whom we have worked or who have published literature on children's mental health services implementation and ripple effects. Approximately 120 policy makers were invited to participate; while most did not respond, some stated that they could not take part in the research due to conflicts of interest resulting from the monetary incentive. Despite the fact that incentives could be declined or immediately donated to charity, policy makers felt uncomfortable with any sort of public record of having participated in a study that provided incentives.

Research staff received emailed requests to participate from 66 youth and caregivers recruited by Youth MOVE National and FREDLA. A team internal database of potential researchers and providers for behavioral health groups provided an additional 148 individuals we asked if they would be interested in participating via blind email; 136 participants responded they would be interested and were sent a consent form via online survey. Following consent survey reach-outs, 135 gave consent to join the study. All participants were sent survey items to complete individually or by way of guided interview, with study staff receiving 81 complete responses to the survey.

Informed consent occurred online. Participants under 18 years of age were required to obtain signed consent from a parent or legal guardian. They received a $\$ 100$ gift card for completion of this first survey. Study data were collected and managed using REDCap online data capture tools hosted at the University of Washington. ${ }^{39}$ The survey required approximately 30 minutes to 1 hour to complete. Youth participants were offered the opportunity to complete the survey with the support of a telephone or video-enabled interview or live access to a research coordinator to field questions. All but one youth participant chose this option. 
The current manuscript presents the results from one portion of a larger study that will extend this work by linking ripple effects to the most common implementation strategies, and identifying gaps in researchers' ripple effect awareness. This study was determined to be exempt from review by the University of Washington Institutional Review Board.

\section{Measures}

Participants provided gender, race, and ethnicity. Participants then read definitions of EBIs, EBI implementation strategies, and ripple effects, and were provided with three examples of ripple effects. One example read, "Training therapists in an EBI has the intended effect of increasing therapist use of an EBI and quality of treatment for clients. If training is done well, there may be several positive ripple effects: therapists might find increased satisfaction with their job, increased feelings of self-worth in the work they do, and may be less likely to leave their job in search of other opportunities. However, if training is done poorly or is done when therapists are already feeling burdened, it may lead to negative ripple effects: therapists may experience burnout, fatigue, dissatisfaction, resistance to the training, and be more likely to leave their job in search of other opportunities, and their clients may be dissatisfied at poor service." Participants were directed to brainstorm up to 15 ripple effects, and to provide a name and a description or definition for each. To encourage brainstorming, participants were told that for every ripple effect they provided, they would be entered in a drawing for an extra $\$ 100$ gift card.

\section{Analyses}

Qualitative analyses were conducted by four experienced members of the team and proceeded in two steps. First, a partially directed conventional content analysis using group consensus was used to thematically code ripple effects. ${ }^{40}$ We began with a list of ripple effects based on the literature and our expertise during the grant writing process and attempted to code each participant's responses using this list. Responses that were agreed to not be ripple effects as defined above were discarded. Responses that were ripple effects but not in our existing list were added. Using consensus decision making, names and definitions of codes in the existing list were iteratively edited to better fit the data. During this process, several codes were merged with other, highly similar codes. When possible, we coded ripple effects so they did not indicate valence, or merged two opposite valence codes into a code without valence (e.g. "Loss of income" and "Increased income" were merged into "Income"), but this was often not possible (e.g. "Overspecialization in EBIs"). Second, we applied the constant comparative method using group consensus to cluster these many ripple effect codes into a smaller number of meaningful ripple effect categories. We engaged in an iterative process of categorization of codes, editing of codes, and recategorization, all while continually comparing the categories and codes to the existing data. Although several codes could reasonably be placed in more than one category, we assigned each code to a single primary category.

\section{Results}


There were 404 unique responses to the ripple effects question; these were coded into 66 unique ripple effects, which were further grouped into 13 categories. This ratio indicates that analyses achieved thematic saturation. Table 2 provides the full list of categories and ripple effects. General knowledge, skills, attitudes, and confidence about using Evidence Based Interventions included 8 ripple effects of the impact on knowledge, skills, attitudes, and confidence of therapists, clients, caregivers, or others regarding a therapist's use of an EBI. An example quote is "Confidence-feeling self-assured to provide a service because you have had the proper training and supervision."

General job-related ripple effects included 6 ripple effects on the attitudes, feelings, and beliefs about one's job and outcomes associated with those attitudes such as worker retention, job satisfaction, and job burnout. An example quote is, "Decreased turnover-Increasing clinicians' felt sense of effectiveness may decrease the desire to switch jobs."

EBI treatment adherence, fidelity and alignment included 3 ripple effects on the degree to which EBIs are delivered by therapists in a way that is consistent with EBI training. An example quote is, "Where the [client's] data is collected but also meaningfully interpreted in order to implement effective practices."

Gaming the system included 4 ripple effects pertaining to manipulating data or practice to achieve the image of an outcome that is not consistent with the spirit of the practice. An example quote is, "People game their therapies to get more money from clients and from agencies to say they did a more elaborate treatment than they truly did."

Equity and stigma included 3 ripple effects on equity (racial, ethnic, and identity group) specifically related to treatment availability, access, quality, and outcomes, as well as stigma associated with mental health issues. An example quote is, "When localities are mandated to perform EBP there could be a potential for localities with less resources to struggle implementing the EBP compared to localities with more resources."

Shifting roles, role clarity, and task shifting included 2 ripple effects on the degree to which the new intervention requires roles that clarifies or conflicts with existing roles, or modifies role responsibilities. An example quote is, "As a parent who is also a provider/professional (peer provider), it can be difficult to manage boundaries with families and providers. Families can think you are 'friends' and providers can forget that you are a family member."

Economic costs and benefits included 4 ripple effects on any monetary cost or benefit of the use of the implementation strategy. An example quote is, "[Training caregiver and youth as peer supporters] provides income/employment to family members who may have had to quit their 'day jobs' already."

EBI treatment availability, access, participation, attendance, barriers, and facilitators included 8 ripple effects on the availability of an EBI in a region (e.g. whether there is a provider that is trained on the EBI), whether the $\mathrm{EBI}$ is accessible (e.g. whether there is no waiting list or other barriers to accessing the treatment such as insurance coverage), client participation and the actual use of in the $\mathrm{EBI}$, and any barriers or facilitators that support the availability, access, and use of services. An example quote is, 
"Increased access-Offering clients the ability to engage with an EBP in an alternative format may increase access to treatment for many youth who do not wish to come in to an office or do not have the resources to do so."

Clinical process and treatment quality included 7 ripple effects on the process of clinical treatment, including treatment quality, the focus, length, assessment burden, time spent in active treatment, and effects on treatment as usual. An example quote is, "Less time in therapy-Increasing access to an effective treatment may decrease the amount of time clients need to be in the system."

Client engagement, therapeutic alliance, and client satisfaction included 5 ripple effects on the degree to which clients are engaged and participating in the treatment planning and process, feelings of alliance and bond between client and therapist, and level of satisfaction with treatment. An example quote is, "Pessimism-Lack of faith in the system which your client is stuck in."

Clinical organization structure, relationships in the organization, process, and functioning included 9 ripple effects on elements associated with the organization, structure, process, functioning, and relationships within the organization, such as organizational climate, organization's use of resources, supervisory relationships, job applicant pool, and referral rates. An example quote is, "Providers [who are trained in EBP] can become more well known, receive accolades, and be supported communally through better treatments and client success."

Youth client and caregiver outcomes included 5 ripple effects on client thoughts, feelings, behaviors, symptoms, and functioning. An example quote is, "Evidence based treatments that improve client functioning can also improve their performance and retention in school, graduation rates, employment rates, and income."

Use of EBI strategies and insights in one's own life includes 2 ripple effects on using EBI strategies for selfcare and using EBP strategies with friends and family members. An example quote is, "EBPs such as deep breathing are utilized by practitioners [to avoid work burnout]."

Table 2 Compilation of possible ripple effects of children's mental health services implementation strategies 


\section{General knowledge, skills, attitudes, and confidence about using Evidence \\ Based Interventions ( $n=$ 8)}

Confidence/Selfefficacy in using EBIs

Skills in using EBIs

Knowledge of EBIs

Beliefs in provider's competence to use EBIs

Overspecialization in EBIs

Feelings of regret for not having used EBIs with past clients

EBI Intervention fatigue

Positive attitude and supportiveness of EBI

\section{General job- related ripple effects $(n=6)$}

Job satisfaction

Job Burnout

Job Retention

Job Autonomy or Independence
Any impact on the knowledge, skills, attitudes, and confidence of therapists, clients, caregivers, or others regarding the use of an Evidence Based Intervention.

One's belief in their capacity to use an EBI with fidelity.

One's actual ability to use an EBI with fidelity.

Facts and information about EBls.

A perception of one's provider having sufficient knowledge, judgment, and/or skills in using EBIs.

To specialize in one or a few EBIs to such a degree as other EBIs are neglected.

Feeling guilt, remorse, or regret for previously implemented, non-EBI treatment practices.

A feeling of being overwhelmed by replacing or supplementing old knowledge and skills with new knowledge and skills, or from trying to learn and master too many varying EBIs.

Degree of support for and belief in the effectiveness of EBIs; includes the amount of hope for improvement resulting from the EBI.

\section{Attitudes, feelings, beliefs about one's job and outcomes associated with those attitudes such as worker retention, job satisfaction, and job burnout.}

The attitudes clinicians, peer support providers, policy makers, or others have about their job as compared to previous experiences, current expectations, or available alternatives.

Feelings of emotional exhaustion, reduced personal accomplishment, loss of work fulfillment, and reduced effectiveness. stay in their job each year.

A feeling of independence and self-determination; ability to make your own decisions and act mostly on your own. When autonomy decreases, one might feel micromanaged. 
Job Workload/ work burden

Sense of job security

EBI Treatment adherence, fidelity, and alignment $(n=3)$

Provider EBI treatment fidelity

Provider mixing of practice elements or cherry-picking practices

Provider using "off-label"

treatment

\section{Gaming the} system $(n=4)$

Data gaming

Treatment counterfeiting

Tokenism

Insufficient use of data tracking

systems

\section{Equity and stigma} $(n=3)$

Racial/ethnic prejudice

Equity

Stigma around mental health issues
Feelings of burden associated with the amount or difficulty of one's work

Feeling that the possibility of losing one's job is very low.

\section{The degree to which EBIs are delivered by therapists in a way that is consistent}

\section{with $\mathrm{EBI}$ training.}

Whether an EBI intervention is implemented as planned and each component is delivered with competence.

Using elements from multiple EBIs in a single practice, incorporating non-EBI elements (for example, dream analysis) into treatment, or using only practices deemed more useful or easier (e.g. teaching relaxation techniques).

Implementing a mental health intervention for a disorder that it was not originally designed to treat. Using a treatment that the clinician knows how to do rather than the treatment most appropriate for the client.

\section{Manipulating data or practice to achieve the image of an outcome that is not consistent with the spirit of the practice.}

Manipulating a data tracking, client management, or progress rewards system for a desired outcome.

Delivering a treatment that is supposed to be a particular EBI but in reality lacks the essential elements of the EBI.

Recruiting people from underrepresented groups to provide feedback or be engaged as advisory board members solely to give the appearance of equality and engagement.

Providers only entering the minimum amount of data necessary to ensure payment or client tracking, and not using the data system as intended.

Equity related to treatment availability, access, quality, and outcomes by race, ethnicity, or other identity group classification; stigma associated with mental health.

\section{Shifting roles, role clarity, task \\ The degree to which the new intervention requires roles that clarifies or conflicts with existing roles, or changes role responsibilities.} shifting $(n=2)$

Being seen as one's racial or ethnic identity rather than individuals.

Role Clarity

Reduction or elimination of disparities (e.g., access to services) between groups stemming from reduction of biases, removal of barriers, and/or inclusion of diverse perspectives.

Stereotypes or negative views attributed to a person or groups of people when their mental health and/or behaviors are viewed as different from or inferior to societal norms.

The degree to which the new intervention clarifies and distinguishes between 
existing roles, tasks, responsibilities, and processes. The opposite of role conflict, blurred roles, or turf battles.

Task shifting When some mental health treatment tasks that had previously been done by therapists are assigned to health workers, peer providers, or others with shorter training and fewer academic qualifications.

Economic costs and benefits $(n=$ 4)

Income

Ability to bill insurance for an

$\mathrm{EBI}$

\section{Budget}

implications

Monetary costs

\section{EBI treatment availability, access, participation, attendance, barriers \& facilitators $(n=8)$}

Service availability, access, and reach

Facilitators of treatment

Barriers to treatment

\section{Any monetary cost or benefit of the use of the implementation strategy.}

Amount of money one makes at one's job.

The ability of therapists to bill insurance plans for a particular EBI or mental health service.

Expected impacts on income and expenditures for agencies or state public mental health budgets.

Monetary costs of the implementation strategy.

The availability of an EBI in a region (e.g. whether there is a provider that is trained on the EBI), whether the EBI is accessible (e.g. whether there is no waiting list or other barriers to accessing the treatment), the actual use of and client participation in the EBI, and any barriers or facilitators that support the availability, access, and use of services

The presence or availability of services and spread of those services across a region-this requires a provider who is trained on the EBI.

Something that facilitates, makes easier, or supports a client's ability to attend, engage in or complete treatment. Facilitators can be at the client level, logistic, or other facilitators. Client-level facilitators might be attitudes (belief treatment will work, belief in the need for treatment), emotional, or behavioral strengths in individuals and groups. Logistic facilitators to treatment include things such as insurance coverage, good transportation, and proximity to treatment.

Something that restricts, impedes, or blocks client ability to attend, engage, or complete treatment. Barriers can be at the client level, logistic, or other barriers. Client-level barriers might be attitudes (stigma, feeling treatment might not work), emotional, or behavioral limitations in individuals and groups. Logistic barriers to treatment include things such as cost, transportation issues, and location.

The degree to which the youth, client, or caregiver is involved in the client's treatment sessions.

Participation or attendance at treatment sessions

Client adherence to treatment recommendations

The degree to which the behavior of youth clients or their caregivers corresponds with recommendations from therapists.

Client transfer/therapist change
Burden and/or instability felt by clients, families, and organizations resulting from changing providers. 
De-

implementation

Clinical process

and treatment quality $(n=7)$

Treatment quality and effectiveness
Sustainability

When a clinical practice or approach to treatment (EBI or non-EBI) that was once offered is discontinued.

Percentage of implemented EBls that sustain at yearly intervals.

The process of clinical treatment, including treatment quality, the focus, length, assessment burden, time spent in active treatment, and effects on treatment as usual.

The degree to which the treatment is high-quality, meaning how effective, engaging, and efficient the treatment is at improving client functioning and symptoms.

Individualization of treatment goals and practice

Length of time until treatment completion

Narrow focus on outcomes

Tailoring treatment goals and practices to the specific needs of the client.

The length of time for a youth client to complete treatment.

Focusing treatment only on one major outcome, neglecting other needs the youth client may have.

Assessment burden

Perception of burden (e.g., hardship or distress) when administering or completing clinical assessments, surveys, and questionnaires.

Time providing or receiving active treatment

Amount of time a provider spends delivering or a client spends receiving active treatment during a session.

Improvement in quality of "usual treatment"

Client engagement, therapeutic alliance, and client satisfaction $(n=5)$

Client engagement in treatment

Clarity of understanding about client's needs and strengths, treatment purpose, and progress

Satisfaction with treatment

Youth client motivation/self-
Improved quality and effectiveness of "treatment-as-usual", usual care, or nonEBI treatment.

The degree to which clients are engaged and participating in the treatment planning and process, feelings of alliance and bond between client and therapist, and level of satisfaction with treatment.
The degree to which the client is engaged in treatment, such as participating during the session, being involved in treatment planning, and initiating contact or questions with the provider.
The degree to which clinicians, clients, or their caregivers are informed about and understand the client's needs and strengths, the reasons for and goals of treatment, and the youth client's clinical progress.
A client's rating of important attributes of the process and outcomes of their treatment experience.
Refers to a youth client's motivation and belief in their capacity to execute behaviors necessary to complete treatment. This reflects confidence in the ability 
efficacy to accomplish

treatment goals alliance
Therapeutic

to exert control over one's own motivation, behavior, and social environment.

A cooperative working relationship between youth client and therapist. Includes youth's sense of being "heard" and responded to in therapy, shared understanding, shared goals, and working together for common therapeutic purpose.

\section{Clinical}

organization

structure,

relationships in

the organization,

process, and

functioning $(n=$

9)

Administrative

burden

\section{Elements associated with the organization, structure, process, functioning, and relationships within the organization, including organizational climate, organization's use of resources, supervisory relationships, job applicant pool, and referral rates.}

Organization

reputation

\section{Organizational}

climate and

culture

Referral rates

Quality of job applicant pool

Opportunity costs

Supervisor-

therapist alliance

Scalability

Cross-

organizational

camaraderie

Youth client and caregiver

outcomes $(n=5)$

Functioning and symptoms
Anything that is necessary to demonstrate compliance with a regulatory requirement, including the collecting, processing, reporting, and retaining of information, and the financial and economic costs of doing so.

Refers to people's collective opinion regarding the organization.

Employee's shared beliefs and values of an organization, and perceptions of the organization's policies, practices, procedures, and reward systems.

The rate of referrals to mental health treatment.

Having a pool of qualified clinician applicants during the hiring process.

The loss of potential gain from other alternatives when one alternative is chosen.

A partnership between a clinical supervisor and a clinician, devoted to the learning and growing of the clinician where there is a strong bond of care, respect, and trust.

An attribute that describes the ability of a process, intervention, or organization to grow and manage increased demand.

Mutual trust, support, friendship, and feelings of community among people.

Client and caregiver thoughts, feelings, behaviors, symptoms, and functioning.

How the client is functioning in the world, and the symptoms of their mental health problems. This could include symptoms like depression, anxiety, or unwanted thoughts, or functioning such as ability to care for themselves, incarceration/recidivism, school enrollment, graduation, out-of-home placements, employment, lifespan, etc.

Informal support systems

Informal support systems such as friends, family, religious supports, recreation groups, and online social networks. 

results from providing prolonged care.

Empowerment

The degree to which one can represent or advocate for their interests, determine the progress of their mental health treatment, and claim their rights.

Re-traumatization Clinical regression (worsening of symptoms or functioning) or new mental health crisis due to processing past traumas.

Hope Hopefulness for the future, inspiration, a belief that the situation will improve.

Use of EBI strategies and The application of strategies and practices from an EBI in one's own life. insight in one's own life $(n=2)$

Using EBI strategies for selfcare

Using EBI strategies with friends and family members
Using EBI strategies in one's own life (such as deep breathing or behavioral activation).

Helping friends and families by teaching them EBI strategies (such as deep breathing or behavioral activation), or using strategies with them such as reward systems.

\section{Discussion}

This study aimed to develop a compilation of possible ripple effects that may result from the implementation of evidence-based children's mental health services. Participants included a diverse array of experienced implementation experts from multiple roles, including youth mental health consumers, caregivers of child and youth consumers, mental health providers, policy makers, and researchers. We identified 66 ripple effects classified into 13 categories.

Given the importance of developing causal conceptual frameworks for implementation efforts, ${ }^{15-18}$ this ripple effects compilation can provide implementers, researchers, funders, policy makers, and consumers with a "menu" of potential ripple effects. Those that are logically or theoretically associated with the implementation efforts can be monitored to ameliorate negative ripple effects and capitalize upon positive ripple effects. This research can serve to increase understanding of the complexity of implementation efforts and the recognition that implementation of EBls is but one action in a larger, interconnected system. We caution that this compilation should not be used as a checklist of all areas to be included in implementation research and quality control efforts. However, we encourage it to be used before and during implementation efforts as a list of possible ripple effects to consider when evaluating the full balance of implementation. This list can be a practical tool to help researchers and implementers be consistent with guidance to explore for unexpected outcomes from experts in QI/QA, Complexity Science, and Diffusion of Innovation Theory, as well as the Medical Research Council. ${ }^{21,22,24-26}$

We also hope this compilation can serve to normalize and elevate the discussion of ripple effects. As discussed, ripple effects might not currently receive attention because they are out of scope or because of 
innovation bias prevents their identification. When ripple effects are identified, they might also be minimized when they are contradictory to the expectations of researchers and implementers.

Implementation efforts often do not go as planned. Tunnel vision impacts both what is investigated and what is reported, ${ }^{41}$ doubly reducing the chances that ripple effects are discussed. Regarding negative ripple effects, the pressures of funders' priorities, political agendas, and career goals may sometimes (consciously or otherwise) override rational, open scientific investigation and reporting. This may be even more true when an implementation effort has positive primary effects (e.g. improved client mental health functioning) and negative ripple effects (e.g. provider burnout and turnover), and implementers may wish to avoid "tainting" their positive findings by providing a balanced view of the overall implementation effects. By elevating the discussion of ripple effects and recognizing their significance, we hope that exploring for them becomes a system-wide expectation of implementation efforts. Consideration of Ripple Effects might be included in the Standards for Reporting Implementation Studies (StaRI) ${ }^{42}$ or other standards checklists, and used when conducting peer review of grants, manuscripts, and conference presentations.

Implementers and researchers may wish for more guidance on which ripple effects are most likely to result from the most common children's mental health implementation strategies, and how to measure them. To partially address this, the next step in the larger project will involve expert participants completing surveys rating which of the ripple effects are most likely to occur for their role, for the implementation strategies rated as most salient to their role. Many of the ripple effects do not have existing measures or, if the measures do exist, they may be lengthy and burdensome. The inclusion of ripple effects measures in a study is, by definition, exploratory. Ripple effects measures may not be included in studies more often due to feasibility and burden. There is a strong need for ripple effects to be quantified using pragmatic measures, that is, measures which are (a) acceptable and relevant to multiple stakeholders, (b) compatible with the setting, (c) short and easy to administer, and (d) useful for decision-making. ${ }^{43,44}$ Unfortunately, developing measures is a time-consuming process, and in the case of ripple effects, may require scenarios that rarely occur. In the coming phases of the study, we will use a participatory research approach with Youth MOVE National in order to refine and test an existing process for developing pragmatic measures using a vignette-based approach, ${ }^{38}$ which eases the burden of measurement development.

\section{Limitations}

There are several limitations to generating this compilation. First, as in any study, a different participant pool may have yielded different results. Our participant base was limited to the United States by participants with implementation expertise who may have had more positive attitudes towards EBPs than general people in their respective roles. By broadening our pool to include multiple roles, we hoped to develop a relatively comprehensive participant base, but this may have also reduced the number of participants with expertise in implementation strategies. Our participation rate from policy makers was much lower than desired, likely due to the difficulty in incentivizing government workers because of rules against providing monetary incentives, and time pressures due to COVID-19. Second, it was difficult to balance parsimony with comprehensiveness during the coding and classification process. While we 
attempted to create valence-free codes, this was often not possible, as some codes did not have clear opposites (e.g. "burnout" may contrast with multiple codes, including job satisfaction, retention, and empowerment, but it is not the opposite of any single code). Third, to manage our resources and obtain input from the largest number of participants, we used online surveys rather than focus groups or in person interviews, with an exception for telephone interviews with youth. While we provided as much supporting documentation as possible, and encouraged participants to contact us with questions, it is possible that participants who are less familiar with differentiating implementation strategies from interventions (and the greyness in between) did not fully understand the survey, ${ }^{45}$ and these difficulties may have impacted the validity of our findings.

\section{Conclusions}

The current study makes a significant contribution to children's mental health services implementation efforts, as well as implementation science in general, by providing a clear and useful compilation of possible ripple effects. Its utility will be expanded in further study phases. We encourage this manuscript to be a starting point for consideration and discussion of ripple effects in general and encourage the development of ripple effect compilations in other fields and areas of implementation. We welcome comments and critiques to add to and iterate upon this compilation to benefit a greater understanding of implementation efforts and their effects.

\section{Abbreviations}

EBI - Evidence Based Intervention

FREDLA - Family-Run Executive Director Leadership Association

$\mathrm{MH}$ - Mental Health

NASMHPD - National Association of State Mental Health Program Directors

SIRC - Society for Implementation Research Collaboration

SMART Center - School Mental Health Assessment and Training Center

Youth MOVE - Youth Motivating Others through Voices of Experience

QI/QA - Quality Improvement/Quality Assurance

\section{Declarations}

\section{Ethics declarations}

\section{Ethics approval and consent to participate}


The Secondary Outcomes Study (STUDY00006331) was reviewed by the University of Washington Institutional Review Board and determined to be exempt from review under Category 2. Participant consent was obtained through an electronic consent form with opportunities and contact information for participants to discuss informed consent with study staff.

\section{Consent for publication}

Not applicable.

\section{Availability of data and materials}

The dataset used during the study are available from the corresponding author on reasonable request.

\section{Competing interests}

The authors declare that they have no competing interests.

\section{Funding}

Funding for the project was supported by the National Institute of Mental Health (NIH; R21MH119360). CC was also supported by the Institute of Education Sciences (IES; R305B170021). BJP was also supported by the National Institute of Mental Health through K01MH113806.

\section{Authors' contributions}

MDP led the conception and design of this study, in addition to obtaining funding, identifying research participants, writing the manuscript, and analyzing the data; SD contributed to the conception and design of the study, obtaining funding, identifying research participants, analyzing data, and writing the manuscript; MTD contributed to the conception and design of the study and obtaining funding; ARL contributed to the conception and design of the study, obtaining funding, identifying research participants, and writing the manuscript; IM, CMC, and CJD contributed to writing and editing the manuscript, collecting data, and analyzing data; KT and MS contributed to identifying research participants, writing, and editing the manuscript; CCL and BJP contributed to the conception and design of the study, obtaining funding, and writing the manuscript.

\section{Acknowledgements}

Not applicable

\section{References}

1. Perou R, Bitsko RH, Blumberg SJ, Pastor P, Ghandour RM, Gfroerer JC, et al. Mental health surveillance among children-United States, 2005-2011. Morb Mortal Wkly Rep Suppl. 2013;62(2):1-35. 
2. Bruns EJ, Walker JS, Bernstein A, Daleiden E, Chorpita BF. Family voice with informed choice: coordinating wraparound with research-based treatment for children. :15.

3. Chorpita BF, Daleiden EL, Bernstein AD. At the intersection of health information technology and decision support: measurement feedback systems...and beyond. Adm Policy Ment Health Ment Health Serv Res. 2016 May 1;43(3):471-7.

4. Bruns EJ. How research-based is our policy-making? Implementation of evidence-based treatments by state behavioral health systems, 2001-2012.:1.

5. Weisz JR, Jensen-Doss A, Hawley KM. Evidence-based youth psychotherapies versus usual clinical care. Am Psychol. 2006;19.

6. Proctor EK, Powell BJ, McMillen JC. Implementation strategies: recommendations for specifying and reporting. Implement Sci IS. 2013 Dec 1;8:139.

7. Bunger AC, Powell BJ, Robertson HA, MacDowell H, Birken SA, Shea C. Tracking implementation strategies: a description of a practical approach and early findings. Health Res Policy Syst. 2017 Feb 23;15(1):15.

8. Rudd BN, Davis M, Beidas RS. Integrating implementation science in clinical research to maximize public health impact: a call for the reporting and alignment of implementation strategy use with implementation outcomes in clinical research. Implement Sci IS. 2020 Nov 25;15(1):103.

9. Boyd MR, Powell BJ, Endicott D, Lewis CC. A method for tracking implementation strategies: an exemplar implementing measurement-based care in community behavioral health clinics. :13.

10. Proctor E, Silmere H, Raghavan R, Hovmand P, Aarons G, Bunger A, et al. Outcomes for implementation research: conceptual distinctions, measurement challenges, and research agenda. Adm Policy Ment Health. 2011;12.

11. Powell BJ, Waltz TJ, Chinman MJ, Damschroder LJ, Smith JL, Matthieu MM, et al. A refined compilation of implementation strategies: results from the expert recommendations for implementing change (ERIC) project. Implement Sci. 2015 Dec;10(1):21.

12. Waltz TJ, Powell BJ, Matthieu MM, Damschroder LJ, Chinman MJ, Smith JL, et al. Use of concept mapping to characterize relationships among implementation strategies and assess their feasibility and importance: results from the expert recommendations for implementing change (ERIC) study. Implement Sci. 2015 Dec;10(1):109.

13. Cook CR, Lyon AR, Locke J, Waltz T, Powell BJ. Adapting a compilation of implementation strategies to advance school-based implementation research and practice. Prev Sci Off J Soc Prev Res. 2019 Aug;20(6):914-35. 
14. Lyon AR, Cook CR, Locke J, Davis C, Powell BJ, Waltz TJ. Importance and feasibility of an adapted set of implementation strategies in schools. J Sch Psychol. 2019 Oct;76:66-77.

15. Powell BJ, Beidas RS, Lewis CC, Aarons GA, McMillen JC, Proctor EK, et al. Methods to improve the selection and tailoring of implementation strategies. J Behav Health Serv Res. 2017 Apr;44(2):177-94.

16. Franks RP, Bory CT. Strategies for developing intermediary organizations: considerations for practice. 98(1):8.

17. Grol R, Bosch M, Wensing M. Development and selection of strategies for improving patient care. 2013;165-84.

18. Lewis CC, Klasnja P, Powell BJ, Lyon AR, Tuzzio L, Jones S, et al. From classification to causality: advancing understanding of mechanisms of change in implementation science. Front Public Health. 2018 May 7;6:136.

19. Mannion R, Braithwaite J. Unintended consequences of performance measurement in healthcare: 20 salutary lessons from the English National Health Service: consequences of performance measurement. Intern Med J. 2012 May;42(5):569-74.

20. Merton RK. Social theory and social structure. 1968 enlarged edition. New York: New York: Free Press; 1968.

21. Rogers EM. A prospective and retrospective look at the diffusion model. J Health Commun. 2004 Jan;9(sup1):13-9.

22. Brainard J, Hunter PR. Do complexity-informed health interventions work? A scoping review. Implement Sci. 2015 Dec;11(1):127.

23. Park AL, Tsai KH, Guan K, Chorpita BF. Unintended consequences of evidence-based treatment policy reform: is implementation the goal or the strategy for higher quality care? Adm Policy Ment Health Ment Health Serv Res. 2018 Jul;45(4):649-60.

24. Moore GF, Audrey S, Barker M, Bond L, Bonell C, Hardeman W, et al. Process evaluation of complex interventions: Medical Research Council guidance. BMJ. 2015 Mar 19;350(mar19 6):h1258-h1258.

25. Toma M, Dreischulte T, Gray NM, Campbell D, Guthrie B. Balancing measures or a balanced accounting of improvement impact: a qualitative analysis of individual and focus group interviews with improvement experts in Scotland. BMJ Qual Saf. 2018 Jul;27(7):547-56.

26. Science of improvement: establishing measures | IHI - Institute for Healthcare Improvement [Internet]. [cited 2020 Nov 23]. Available from:

http://www.ihi.org:80/resources/Pages/Howtolmprove/ScienceofImprovementEstablishingMeasures.aspx 
27. Sveiby K-E, Gripenberg P, Segercrantz B, Eriksson A, Aminoff A. Unintended and undesirable consequences of innovation. :16.

28. Jones EL, Lees N, Martin G, Dixon-Woods M. How well is quality improvement described in the perioperative care literature? A systematic review. Jt Comm J Qual Patient Saf. 2016 May;42(5):196-AP10.

29. Nicolay CR, Purkayastha S, Greenhalgh A, Benn J, Chaturvedi S, Phillips N, et al. Systematic review of the application of quality improvement methodologies from the manufacturing industry to surgical healthcare. Br J Surg. 2012 Mar;99(3):324-35.

30. Manojlovich M, Lee S, Lauseng D. A systematic review of the unintended consequences of clinical interventions to reduce adverse outcomes. J Patient Saf. 2016 Dec;12(4):173-9.

31. Forman-Hoffman VL. Quality improvement, implementation, and dissemination strategies to improve mental health care for children and adolescents: a systematic review. 2017;21.

32. Lester H, Birchwood M, Freemantle N, Michail M, Tait L. REDIRECT: cluster randomised controlled trial of GP training in first-episode psychosis. Br J Gen Pract. 2009 Jun 1;59(563):e183-90.

33. Aarons GA, Sommerfeld DH, Hecht DB, Silovsky JF, Chaffin MJ. The impact of evidence-based practice implementation and fidelity monitoring on staff turnover: evidence for a protective effect. J Consult Clin Psychol. 2009 Apr;77(2):270-80.

34. Aarons GA, Fettes DL, Flores LE, Sommerfeld DH. Evidence-based practice implementation and staff emotional exhaustion in children's services. Behav Res Ther. 2009 Nov;47(11):954-60.

35. Ryan AM, McCullough CM, Shih SC, Wang JJ, Ryan MS, Casalino LP. The intended and unintended consequences of quality improvement interventions for small practices in a community-based electronic health record implementation project. Med Care. 2014 Sep;52(9):826-32.

36. Merton RK. The unanticipated consequences of purposive social action. Am Sociol Rev. 1936 Dec;1(6):894.

37. Silver N. The signal and the noise: why so many predictions fail-but some don't. New York: New York: Penguin Press; 2012.

38. Weiner BJ, Lewis CC, Stanick C, Powell BJ, Dorsey CN, Clary AS, et al. Psychometric assessment of three newly developed implementation outcome measures. Implement Sci. 2017 Aug 29;12(1):108.

39. Harris PA, Taylor R, Minor BL, Elliott V, Fernandez M, O’Neal L, et al. The REDCap Consortium: building an international community of software platform partners. J Biomed Inform. 2019 Jul;95:103208.

40. Strauss AL, Glaser BG. The discovery of grounded theory: strategies for qualitative research. Chicago: Chicago: Aldine Publishing; 1967. 
41. Rosenthal R. The file drawer problem and tolerance for null results. Psychol Bull. 1979;86(3):638-41.

42. Pinnock H, Barwick M, Carpenter CR, Eldridge S, Grandes G, Griffiths CJ, et al. Standards for reporting implementation studies (StaRI) statement. BMJ. 2017 Mar 6;356:i6795.

43. Powell BJ, Stanick CF, Halko HM, Dorsey CN, Weiner BJ, Barwick MA, et al. Toward criteria for pragmatic measurement in implementation research and practice: a stakeholder-driven approach using concept mapping. Implement Sci. 2017 Dec;12(1):118.

44. Pullmann MD, Ague S, Johnson T, Lane S, Beaver K, Jetton E, et al. Defining engagement in adolescent substance abuse treatment. Am J Community Psychol. 2013 Dec;52(3-4):347-58.

45. Eldh AC, Almost J, DeCorby-Watson K, Gifford W, Harvey G, Hasson H, et al. Clinical interventions, implementation interventions, and the potential greyness in between -a discussion paper. BMC Health Serv Res. 2017 Jan 7;17(1):16.

\section{Supplementary Files}

This is a list of supplementary files associated with this preprint. Click to download.

- 04262SRQRChecklist.docx 